\title{
TAVI safe and effective for patients with a bicuspid aortic valve
}

Patients with a bicuspid aortic valve (BAV) can safely and successfully undergo transcatheter aortic valve implantation (TAVI). The condition was previously thought to be a counterindication for TAVI, because of the risk of uneven expansion of the bioprosthesis. BAV is a congenital condition that affects $0.5-2.0 \%$ of the population, so this new finding might increase the number of patients, particularly those of a young age, who are eligible for TAVI.

Investigators from the Institut Cardiovasculaire Paris Sud, France, analysed data from 229 consecutive patients from their TAVI database who had undergone both echocardiography and multidetector computed tomography (MDCT). BAV was detected in $9.2 \%$ of patients using MDCT, but in only $3.9 \%$ using transthoracic and transoesophageal echocardiography, indicating that BAV is underdetected using echocardigraphy compared with MDCT.

Patients received an Edwards SAPIEN ${ }^{\circledR}$ valve (Edwards Lifesciences Corporation, Irvine, CA, USA) or a CoreValve (Medtronic CV Luxembourg S.a.r.l., Luxembourg). In patients with a tricuspid aortic valve, the Edwards valve was used more often than the Medtronic valve ( $83.7 \%$ vs $16.3 \%)$, but no difference existed in the frequency of use of the two types of valve in patients with a BAV (52.4\% vs $47.6 \%$ ).

The incidence of periprocedural complications did not significantly differ according to valve morphology, and no annulus rupture or valve migration was observed in patients with a BAV. Functional outcomes were similar between the two groups, and no significant difference existed in the incidence of postprocedural aortic regurgitation grade 2 or higher. Neither 30-day mortality nor the incidence of the 30-day combined safety end point differed significantly according to whether two or three aortic valve leaflets were present.

These data come from a retrospective, single-centre study with a small cohort size. Nevertheless, they indicate that TAVI might be similarly safe and effective in patients with a BAV as in those with a tricuspid aortic valve, regardless of the type of prosthetic valve used. Further studies are needed in larger populations of patients.

Gregory B. Lim 